\title{
On global stability of thin ionized disks immersed in an external magnetic field
}

\author{
Edward Liverts and Michael Mond \\ Department of Mechanical Engineering, Ben-Gurion University of the Negev, \\ P.O. Box 653, Beer-Sheva 84105, Israel \\ email: eliverts@bgu.ac.il, mond@bgu.ac.il
}

\begin{abstract}
The problem of the global stability of rotating magnetized thin disks is considered. The appropriate boundary value problem (BVP) of the linearized MHD equations is solved by employing the WKB approximation to describe the dynamical development of an initial perturbation. The eigenfrequencies as well as eigenfunctions are explicitly obtained and are verified numerically. The importance of considering the initial value problem (IVP) as well as the question of global stability for finite systems is emphasized and discussed in detail. It is further shown that thin enough disks are stable (global stability) but as their thickness grows increasing number of unstable modes participate in the solution of the IVP. However it is demonstrated that due to the localization of the initial perturbation the growth time of the instability may be significantly longer than the calculated inverse growth rate of the individual unstable eigenfunctions.
\end{abstract}

Keywords. Accretion disks - magnetic fields - MHD - instabilities

We consider geometrically thin disks and weak magnetic fields $V_{A}<c_{s}$ where $V_{A}=$ $B_{z} / \sqrt{4 \pi \rho_{0}}$ is the Alfvén velocity and $c_{s}$ is the isothermal sound velocity. In order to model the finite thickness of the disk the following profile $\rho(r, z)=\rho_{0}(r) \exp \left(-z^{2}\right)$ is assumed for the density (the disk is assumed to be isothermal). It is assumed that the typical length scale for the variation of the physical quantities in the radial direction is much longer than that in the vertical direction. For that reason the disk model incorporates the vertical stratification while the radial structure of the disk ignored excepting of the velocity shear. Thus, due to the independence of the perturbations on the radial direction $r$ is merely a parameter. It should be noted however that in the classical works of Velikhov (1959), Chandrasekhar (1960), and Balbus \& Hawley (1991), an infinite cylinder has been considered and the effects of the boundary conditions as well as vertical structure of the disk were neglected. Therefore, in those works, naturally, the thickness of the disk does not play any role and consequently cannot influence the extent of the domains of instability.

To perform the stability analysis of such stratified disks the common practice of taking the Laplace transform for arbitrary initial conditions (IC), consequently reducing the set of linear equations describing dynamical system to the single inhomogeneous ordinary differential equation (ODE), and solving the latter subject to certain boundary conditions (BC), is employed [see for example the well-known work of Landau (1946)].

The $\mathrm{BC}$ of the considered problem are obtained due to the requirement that at $z \rightarrow$ $\pm \infty$, where $\rho \rightarrow 0$ and $B_{z}=$ const, the energy flux of the perturbation is finite.

The solution of such problems is facilitated by obtaining the natural frequencies of the bounded system $\omega_{n}$ for which the state vector of the linearized dynamical system may be written as $\mathbf{u}_{\mathbf{n}}(z, t)=\mathbf{A}_{n}(z) e^{-i \omega_{n} t}$, where $\mathbf{A}_{n}(z)$ are the eigenfunctions of the BVP subjected to specific BC. See also Sano \& Miyama (1999), Coppi \& Coppi (2001), Coppi \& Keyes (2003). 

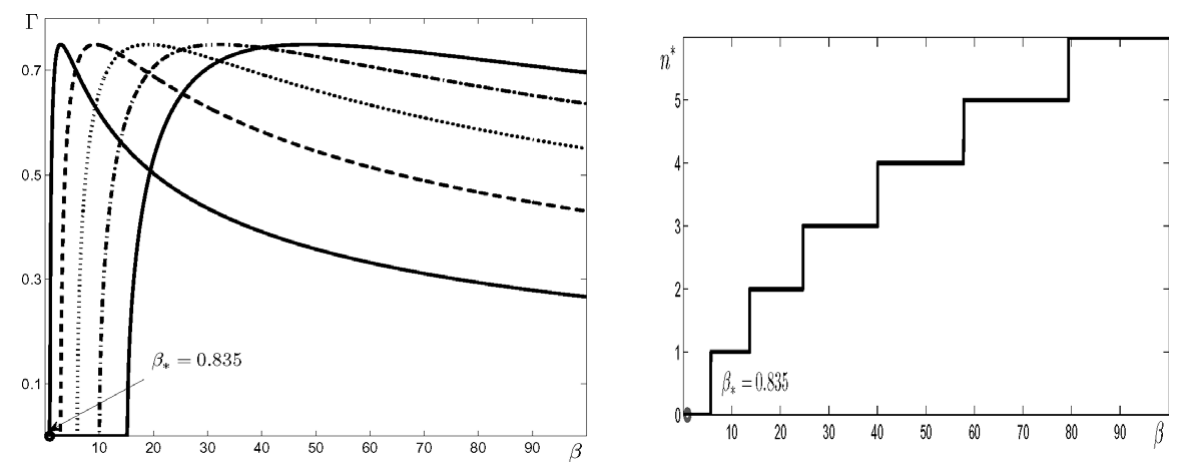

Figure 1. Left panel. Growth rate $\Gamma$ vs plasma beta parameter for the first five eigenfunctions $(n=0, . ., 4)$. Right panel. Axial standing wave number of the most unstable mode as a function of the plasma beta.

The full solution of the dynamical evolution in time of a localized Gaussian wave packet using such techniques has been examined recently by Liverts \& Mond 2008.

We summarize our results as follows.

- It has been shown analytically and numerically that in the case of sufficiently thin disk in the sense of the disk thickness $c_{s} / \Omega$ and the Alfvén length $V_{A} / \Omega$ ratio which are defined also by the plasma beta $2 c_{s}^{2} / V_{A}^{2}\left(\beta<\beta_{*}\right.$ Fig. 1$)$, there is no instability at all and the disk is globally stable. It is instructive to note that the importance of studying the influence of the finite size of the system on its stability has been demonstrated by Budker (1956), Sturrock (1958) who reported that the size of system plays a stabilizing role for the two stream instability. If the disk is thin enough, then the localized initial perturbations do not grow in time, they just move back and forth with the local Alfvén velocity (Liverts \& Mond 2008).

- If the thickness of the disk increases $\left(\beta>\beta_{*}\right.$ Fig. 1,left panel) so does the number of unstable modes. Asymptotic estimation reveals that $n^{*}$ (Fig. 1,right panel) is proportional to $\sqrt{\beta}$. This implies that the wavelength of the most unstable mode is of the order of $h / n^{*} \sim V_{A} / \Omega$ (where $h$ is the thickness of the disk).

- Considering the boundary effects may have a large impact on the design of laboratory experiments (Couette flows) to model MRI. See Noguchi et al. (2002), Rüdiger et al. (2006), Ji et al. (2006).

\section{References}

Balbus, S. A. \& Hawley, J. F. 1991, ApJ 376, 214

Budker, G. I. 1956, Atomic Energy 1, 673

Chandrasekhar, S. 1960, Proc. Nat. Acad. Sci. A46, 223

Coppi, B. \& Coppi, P. S. 2001, Phys. Rev. Lett. 87, 051101

Coppi, B. \& Keyes, E. A. 2003, ApJ 595, 1000

Ji, H. T., Burin, M., Schartman, E., \& Goodman, J. 2006, Nature 444, 343

Landau, L. D., 1946, Zh. Eksp. Teor. Fiz. 16, 574 [1946, J. Phys. USSR, 10,25]

Liverts, E. \& Mond, M. 2008, MNRAS (in press), astro-ph 0810.1864v1

Noguchi, K., Pariev, V. I., Colgate, S. A., Beckley, H. F., \& Nordhaus, J. 2002, ApJ 575, 1151

Rüdiger, G., Hollerbach, R., Stefani, F., Gundrum, T., Gerbeth, G., \& Rosner, R. 2006, ApJ $649, \mathrm{~L} 145$

Sano, T. \& Miyama, S. M. 1999, ApJ 515, 776

Sturrock, P. A. 1958, Phys. Rev. A 112, 1488

Velikhov, E. P. 1959, Zh. Eksp. Teor. Fiz. 36, 1398 [1959, Sov. Phys. JETP 36, 995]. 